\title{
UNA REFLEXIÓN CRÍTICA SOBRE LA TRASPOSICIÓN DE LA POLÍTICA URBANA DE LA UNIÓN EUROPEA AL MARCO ESPAÑOL
}

\author{
Sonia de Gregorio Hurtado (Dra. Arquitecta - Investigadora DUyOT)
}

En España al iniciar la década de los ochenta del siglo XX, se inicia la transferencia de competencias a las Comunidades Autónomas. Al finalizar los setenta se habían transferido a los Ayuntamientos. Ambos niveles de gobierno recibieron competencias en el ámbito del urbanismo y otras políticas urbanas, mientras que el Gobierno Central mantuvo muy pocas.

Esto dio lugar a una situación que rompía con la centralidad con la que la acción sobre la ciudad se había ejercido durante la dictadura. Se iniciaba una nueva etapa en la que Comunidades Autónomas y Ayuntamientos tuvieron que hacer frente a la difícil situación en la que se encontraban las ciudades después de un periodo caracterizado por la inacción para mejorar el medio urbano y la falta de reflexión sobre la ciudad. Los expertos y técnicos que estaban activos en el ámbito del urbanismo en ese periodo refieren la ilusión con la que los planificadores y las nuevas corporaciones municipales acometieron esta tarea, lo cual permitió la integración de la cultura urbanística en los nuevos instrumentos de planificación. También señalan el papel que jugó en la mejora urbana la participación ciudadana a través de los Movimientos Sociales Urbanos.

Así pues el inicio de la Transición conllevó la devolución de las competencias al nivel local, llevando la toma de decisiones a los niveles de gobierno que eran los directamente afectados por las políticas urbanas, y acercando el nivel de decisión a los ciudadanos, facilitando la integración de la participación en los procesos.

Desde el punto de vista de la gobernanza multi-nivel el momento se caracterizó a grandes rasgos por la colaboración entre los tres niveles de gobierno. En este sentido experiencias como la Remodelación de Barrios en Madrid (1979-1996) ejemplifican como fue posible la colaboración entre los mencionados tres niveles en un clima de cooperación y apoyo mutuo. También es un ejemplo de como la movilización ciudadana fue clave (dio inicio a la iniciativa), logrando introducir un problema urbano a resolver en la agenda de las nuevas instituciones.

Al finalizar la década de los ochenta y el inicio de los noventa el clima de colaboración multi-nivel perdió intensidad. Esta situación fue común también a otros ámbitos de política en los que las Comunidades y los Ayuntamientos empezaron a funcionar en base a una lógica de defensa de los ámbitos competenciales, dejando insuficientemente atendida la concertación entre los diferentes niveles de gobierno, y alejándose de la colaboración voluntaria entre administraciones que es parte esencial del Estado de las Autonomías. Este hecho explica en gran medida por qué España no abordó a lo largo de la Transición una reflexión a escala de país sobre la ciudad, ya que la falta de competencias del Gobierno Central sumada a las sentencias del Tribunal Constitucional, que declararon inconstitucionales algunos intentos del primero por actuar de manera concertada en ámbitos como la planificación territorial 
o el transporte, fueron "deslegitimando" la acción a escala nacional, de manera que, al contrario que muchos estados de la UE, España no logró dotarse de una política urbana a nivel de país y el Gobierno Central quedó desdibujado como actor en relación a este ámbito de acción política. El Plan Director de Infraestructuras de 1993 fue el intento más avanzado del Gobierno Central por actuar en el medio urbano en un clima de concertación con las Comunidades Autónomas y las ciudades, a través de la llamada Política de Ciudades. Sin embargo en 1996 la misma (que había empezado a dar lugar a resultados positivos) fue suprimida.

También en ese momento la participación empezó a perder vigor. Borja (2001) señala como uno de los factores que frenaron el desarrollo de la democracia local participativa las organizaciones partidarias que tienden al monopolio institucional de la vida política local. Urrutia señala la puesta en práctica de modelos de participación ciudadana con escasa voluntad política para incorporar a las organizaciones ciudadanas. Para él esa circunstancia dio lugar a dos actitudes frecuentes al inicio de la década de los noventa: el exclusivismo (acción de apoyo preferente por parte de las instituciones a determinadas organizaciones más cercanas a la línea política del gobierno); y el paternalismo institucional respecto a los ciudadanos y sus organizaciones "considerando como «favor» aquello que es «derecho» (Urrutia, 1992: 51). Ambas actitudes también describen el modo según el cual se ha venido entendiendo la participación hasta el momento presente en el ámbito del urbanismo en nuestro país, a pesar del desarrollo de valiosas excepciones.

En este contexto, muy simplificado en la exposición por una cuestión de extensión, al finalizar los años 80 e iniciar los años 90 la Comunidad Económica Europea (CEE) empieza a dirigir su atención a las ciudades, al entender que en estos ámbitos se habían concentrado a lo largo de las dos décadas anteriores problemas que limitaban el camino hacia la cohesión de los Estados Miembros (social y económica). A través de un camino complejo las instituciones comunitarias pusieron en marcha un proceso que derivó en la conformación de lo que hoy podemos llamar "política urbana de la Unión Europea (UE)", un ámbito en el que la UE no tiene competencias, formalizado poco a poco a través de un proceso complejo en el que ha tenido un papel importante el lanzamiento de instrumentos de política no vinculantes: comunicaciones de la Comisión Europea y documentos políticos de otros actores relevantes, líneas guía de actuación, instrumentos dotados de contenido económico para la regeneración urbana, intercambio de buenas prácticas, programas de construcción de capacidad y aprendizaje, etc. En 1989 la Comisión lanzó el primer instrumento específico de esta política (los Proyectos Piloto Urbanos -PPU-). Sobre la experiencia del mismo en 1994 se lanzó la Iniciativa Comunitaria URBAN para la regeneración urbana, el principal instrumento de la política urbana de la UE hasta el momento, cuyo desarrollo ha hecho una aportación de primer orden a la práctica de la regeneración en el contexto nacional (De Gregorio, 2012; De Gregorio, 2014). Este último ha asumido en gran medida la metodología de actuación de URBAN, aplicando el concepto de" regeneración urbana integrada" en barrios degradados (ibid.).

Volviendo al marco español, ya durante la segunda mitad de los ochenta y primeros noventa se pusieron de relieve una serie de cuestiones en relación a la actuación en el medio urbano que era importante atender, muchas de ellas eran fruto de la falta de acción del periodo dictatorial, mientras que otras empezaron a tener que ver con la nueva arquitectura institucional y con como empezaba a evolucionar 
la gobernanza asociada a la misma (en su dimensión multi-nivel, horizontal y de participación) y con el momento histórico (la globalización, la retirada del Estado como actor económico, el reto ambiental, la competitividad urbana). Otras, como el desafío demográfico, el cambio climático o la pobreza urbana, se han perfilado en los años siguientes en relación a nuevos problemas políticos, ambientales, sociales y económicos con importantes componentes inherentes a la realidad española: todas ellas cuestiones a abordar desde una reflexión que no se ha dado ni a escala de país, y salvo excepciones, tampoco a escala de las Comunidades Autónomas.

La falta de acción del Gobierno Central y de las Comunidades Autónomas contribuyó a que el primer plano de la reflexión sobre la actuación en la ciudad consolidada en el marco nacional fuera ocupado en gran parte desde el final de los años noventa por la política urbana de la UE. Esto se ha venido haciendo en una clave de trasposición directa de las metodologías y prioridades comunitarias al marco español, lo que ha conseguido desplazar la atención de la especificidad de nuestros temas pendientes y ha relegado la necesidad de abordar la acción en el medio urbano en base a una reflexión estratégica sobre los problemas a resolver, pero también sobre los muchos elementos positivos a preservar que son inherentes al modelo de ciudad en nuestro país. Mientras que la Iniciativa Comunitaria URBAN, y en general la política urbana de la UE, han hecho una aportación positiva a la práctica de la regeneración urbana en España (De Gregorio, 2012 y 2014), la observación de la evolución de su influencia sobre el marco español, una vez casi finalizados los proyectos de la Iniciativa Urbana ${ }^{11}$, empieza a poner de manifiesto que dicha aportación está contribuyendo a mantener en segundo plano nuestra reflexión pendiente (De Gregorio, 2015).

En el periodo 2004-2011 un esfuerzo del Gobierno Central, formalizado en el lanzamiento de documentos como el Libro Verde de Medioambiente Urbano (MARM, 2007 y 2009), la Estrategia Española de Medioambiente Urbano (MARM, 2008) o la Estrategia Española de Sostenibilidad Urbana y Local (MARM, 2011), junto con otras instrumentos de política entre los que cabe destacar el Plan Estratégico de Infraestructuras y Transporte (Ministerio de Fomento, 2005), parecían señalar el inicio de un camino que pretendía devolver al tema urbano atención por parte de las instituciones y de otros actores desde una visión de concertación (De Gregorio, 2012). Sin embargo, la observación del bajo impacto que dichos instrumentos han tenido en la acción de los gobiernos locales y su incapacidad de dar visibilidad al tema y provocar una reflexión sostenida sobre la ciudad, permite decir que se ha tratado de un esfuerzo que no ha llegado a dar resultados, o al menos los resultados esperados, y una vez más demuestra la incapacidad del país de poner esta cuestión en el centro de la atención y la acción política.

Como consecuencia, en el momento actual persisten una serie de cuestiones importantes que han quedado relegadas y desatendidas, invisibilizadas en gran medida por la política urbana de la UE. En este momento se están definiendo los

\footnotetext{
11 La iniciativa Comunitaria URBAN se desarrolló en dos fases: URBAN (1994-1999) y URBAN II (20002006). A partir de 2007 en el marco de la UE se inicia el mainstreaming de la política urbana. Desde ese enfoque países como España deciden dar continuidad a URBAN de manera explícita en su marco nacional a través de las ayudas del Fondo Europeo de Desarrollo Regional (FEDER) para el desarrollo urbano sostenible. En nuestro país esto se ha llevado a cabo a través de los proyectos de la Iniciativa Urbana (2008-2013).
} 
instrumentos que determinarán la implementación de dicha política en España en el periodo presente de la Política de Cohesión (2014-2020). Por esta razón, es clave recuperar esos temas pendientes e introducirlos en la acción en el medio urbano a través de los recursos del Fondo Europeo de Desarrollo Regional (FEDER), sin llevar a cabo una vez más una transposición directa de las directrices y metodologías comunitarias, sino una aplicación de las mismas adaptada y matizada por la realidad en la que actúan. Algunos de esos temas/acciones pendientes en relación a la actuación en el medio urbano que se proponen desde aquí son los siguientes:

- Una reflexión promovida por los Ayuntamientos, las Comunidades Autónomas y el Gobierno Central sobre la ciudad y, por tanto, también sobre el concepto/enfoque de la regeneración urbana que es necesario adoptar. Esta reflexión habría de incluir un debate sobre la gobernanza multi-nivel en relación a la actuación en el medio urbano.

- Abordar una reflexión sobre la sectorialidad desde la que se sigue actuando en la ciudad a través de proyectos de regeneración, a pesar de la creciente utilización del término "regeneración urbana integrada" para denotar muchas de esas actuaciones.

- En relación a lo anterior, dar lugar a una revisión del término de "regeneración urbana integrada", cuyo uso retórico en la trasposición del mismo al marco español desde el contexto comunitario parece haber derivado en un uso "acrítico"12 del mismo.

- Introducción en el concepto de regeneración urbana de temas poco integrados hasta el momento: la belleza urbana, el género, la mitigación y la adaptación al cambio climático, el desafío demográfico, la pobreza urbana, etc.

- Abordar la participación de la comunidad local desde una revisión de los instrumentos de participación previstos por la Ley 57/2003 de Medidas para la Modernización del Gobierno Local, al haberse mostrado estos ineficaces para canalizar la participación efectiva de los ciudadanos y sus organizaciones, así como de otros actores relevantes.

- Reflexionar sobre el insuficiente análisis, seguimiento y evaluación de políticas, ligado al bajo nivel de trasparencia y rendición de cuentas en relación a la actuación en la ciudad. En relación a esto, poner en marcha el Observatorio Urbano que se anunció en 2003 en el marco de la mencionada Ley 57/2003.

\footnotetext{
12 Uso aquí el adjetivo "acrítico" que utiliza José Manuel Naredo en relación al uso del término "desarrollo sostenible" en su texto Sobre el origen, el uso y el contenido del término sostenible, al considerar que existen similitudes en el uso retórico de este término y el de "regeneración urbana integrada". Disponible en: http://habitat.aq.upm.es/cs/p2/a004.html (última consulta 25 de junio de 2015).
} 


\section{REFERENCIAS BIBLIOGRÁFICAS:}

BORJA, J. (2001). "Participación, un desafío, una oportunidad, una cuestión política", en Proyecto Educativo de Ciudad. Gijón, Ayuntamiento de Gijón.

DE GREGORIO HURTADO, S. (2012). Políticas urbanas de la Unión Europea desde la perspectiva de la Planificación Colaborativa. Las Iniciativas Comunitarias URBAN y URBAN II en España. Tesis Doctoral. Escuela Técnica Superior de Arquitectura de la Universidad Politécnica de Madrid.

DE GREGORIO HURTADO, S. (2014). "La Iniciativa Comunitaria URBAN como factor de transformación de la práctica de la regeneración urbana: aproximación al caso español", en Ciudad y Territorio. Estudios Territoriales, volumen XLVI, número 180, pp. 253-276.

DE GREGORIO HURTADO, S. (2015). "La contribución de URBAN y URBAN II a la práctica de la regeneración urbana en España". Presentación en las Jornadas de Política de Regeneración Urbana en España: de las Enseñanzas a los Retos, Ayuntamiento de Lucena: Lucena, 28-29 Enero, 2015.

MARM (Ministerio de Medio Ambiente y Medios Rural y Marino) (2007). Libro Verde de medio ambiente urbano. Tomo I. MARM, Madrid.

MARM (Ministerio de Medio Ambiente y Medio Rural y Marino) (2008). Estrategia española de medio ambiente urbano. MARM, Madrid.

MARM (Ministerio de Medio Ambiente y Medio Rural y Marino) (2009). Libro verde de medio ambiente urbano. Tomo II. MARM, Madrid.

MARM (Ministerio de Medio Ambiente y Medio Rural y Marino) (2011). Estrategia española de sostenibilidad urbana y local. MARM, Madrid.

MINISTERIO DE FOMENTO (2005): Plan estratégico de infraestructuras y transporte. Ministerio de Fomento, Madrid.

URRUTIA, V. (1992): "Transformación y persistencia de los movimientos sociales urbanos, en Política y Sociedad, número 4, pp. 49-56. 